\title{
Load approximation for uncertain topologies in the low-voltage grid
}

\author{
Ludovic Mouline, Maxime Cordy, and Yves Le Traon \\ Interdisciplinary Centre for Security, Reliability and Trust (SnT), \\ University of Luxembourg, Luxembourg, \\ $\{$ first.last\}@uni.lu
}

\begin{abstract}
Smart grids allow operators to monitor the grid continuously, detect occurring incidents, and trigger corrective actions. To perform that, they require a deep understanding of the effective situation within the grid. However, some parameters of the grid may not be known with absolute confidence. Reasoning over the grid despite uncertainty needs the consideration of all possible states. In this paper, we propose an approach to enumerate only valid potential grid states. Thereby, we allow discarding invalid assumptions that poison the results of a given computation procedure. We validate our approach based on a real-world topology from the power grid in Luxembourg. We show that the estimation of cable load is negatively affected by invalid fuse state combinations, in terms of computation time and accuracy.
\end{abstract}

\section{INTRODUCTION}

The embedding of information and communication technology within smart grids enhances grid operations with management systems that can continuously monitor the grid, detect incidents, and trigger healing mechanisms [1], [2]. Such systems rely on (near) real-time knowledge about the grid (e.g., the grid topology and power flow, the customer consumption data sent by smart meters, etc.) and automated ways to interact with it (e.g., fuses that can be controlled remotely). These further pave the way for developing recommender systems to support operations by suggesting relevant actions to perform on the grid [2].

However, the knowledge required to achieve this can be incomplete or inaccurate [3], due to a legacy of unsystematic documentation standards and procedures. This is the case for Creos S.A. (named "Creos" hereafter), our industrial partner, and the energy distributor in Luxembourg. Creos identified a significant number of inaccuracies among collected information related to the low-voltage grid, which is still operated manually. That includes, for instance, missing reports of grid configuration changes (e.g., closing/opening fuses) and also undocumented infrastructure updates (e.g., a new cable was installed but is not encoded in the management system). Because of that, there exist discrepancies between the actual state of the grid (topology and power flow) and the view Creos has on it. That hinders the management systems' ability to produce reliable insights, as their computations (e.g., cable loads) might be substantially affected by erroneous assumptions. For example, operators might suspect a cable is overloading while it is not, or even worse, might overlook an overloading. Fixing documentation inaccuracies is a daunting task that would require manual inspection of a tremendous amount of data. While data quality is a growing concern at Creos and will certainly improve over time, for now, operators have no choice but to deal with uncertainty in their knowledge of the grid.

In this paper, we tackle the problem of computing the electrical loads of the cables in the presence of uncertain knowledge about the states of fuses. Uncertainty on fuses will create different configurations for the same topology. We exploit measured data and domain-specific validity rules to reduce noise in such computations and define an efficient propagation uncertainty. We evaluate the impact of a configuration on the cable load estimation: (RQ1) how much do estimations of cable loads vary in different grid configurations? In our case study, we found out that the highest estimated load can be four times higher than the lowest estimated load. Then, we show that invalid configurations impact the validity of approximated load: (RQ2) do invalid configurations significantly alter the cable load estimations? Moreover, we show that our work contributes to improving reasoning procedures built upon smart grids, in the presence of uncertainty in their topology: (RQ3) what is the performance gained by filtering out invalid grid configurations?

\section{RELATED WORK}

Uncertainty in power grids has been studied since the early '70s [4], [5]. Some studies focused on stochastic input's impacts due to energy generated from renewable sources such as wind or thermal. To tackle this problem, they define approaches to compute the load efficiently. These approaches are referred to as probabilistic optimal power flow (POPF) [6], or, more generally, probabilistic power flow (PPF) [4], [5]. Different approaches have been defined to tackle this problem. For example, authors of [7] proposed a solution using a quasi-Monte Carlo simulation and copula function. [8] described a model that uses the Dirichlet process mixture to solve the challenge of POPF. The dependency between the different stochastic power energy has been studied in [9]. Another uncertainty in the power grid that has been considered is the one affecting 


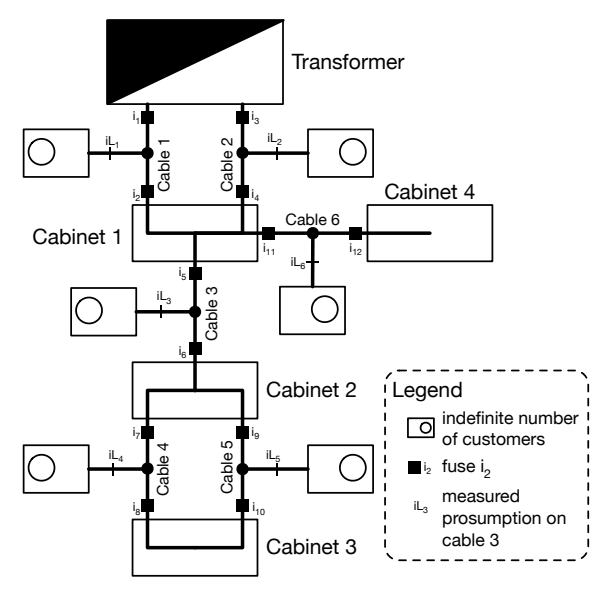

Fig. 1. Schema of the topology used as running example

measurements. Sensed data are affected by measurement errors, measurement precision, and by a loss of data. In [10], the researchers described an approach to address this problem by representing the measurement's confidence level with a complex normal distribution. None of the proposed solutions considers the uncertainty of the grid configuration (fuse states) from the best of our knowledge. [9] classified the PPF approaches into four categories. The classification has been made according to the methodology used: analytical methods, e.g., [11], approximate techniques, e.g., [6], heuristic procedures, e.g., [12], and Monte Carlo simulations, e.g., [7]. We assume that fuse states can be represented by a binary variable. The uncertainty on such elements can be represented by the Bernoulli distribution, and the computation made is simple enough to be done analytically.

\section{Motivation and Running Example}

Figure 1 shows a topology example with one transformer (at the top of the figure) and four cabinets (white rectangles). These entities (transformer and cabinets) are linked through six cables (bold lines). At each end of any cable, there is a fuse noted $i_{n}$, with $1 \leqslant n \leqslant 12$. Each cable has a measured prosumption noted $i L_{k}$, with $1 \leqslant k \leqslant 6$. Among the cables, Cable 1 and Cable 2 are parallel, just like Cable 4 and Cable 5. They thus form two circles. One is composed of fuses $i_{1}, i_{2}, i_{3}$, and $i_{4}$. Fuses $i_{7}, i_{8}, i_{9}$, and $i_{10}$ constitute the other circle.

A fuse is said "uncertain" when its state is not known with absolute confidence. We quantify this uncertainty with a Bernoulli distribution [13]. Each fuse state (closed and open) is attached with a confidence level (probability value) between 0 and 1 . The sum of the confidence levels of each state equals 1 . If $i_{2}$ is uncertain, the operator might know that the fuse is closed with a confidence level of $62 \%$ and open with $38 \%$ confidence.

We define a configuration as a set containing the states of all fuses. For example, $\left\{i_{1}: C, i_{2}: C, i_{3}: C, i_{4}: C, i_{5}\right.$ : $\left.C, i_{6}: C, i_{7}: C, i_{8}: C, i_{9}: C, i_{10}: C, i_{11}: C, i_{12}: C\right\}$, $\left\{i_{1}: C, i_{2}: C, i_{3}: C, i_{4}: C, i_{5}: C, i_{6}: C, i_{7}: O, i_{8}: C, i_{9}:\right.$ $\left.C, i_{10}: C, i_{11}: C, i_{12}: C\right\}$, and $\left\{i_{1}: C, i_{2}: C, i_{3}: C, i_{4}:\right.$ $\left.C, i_{5}: C, i_{6}: C, i_{7}: O, i_{8}: C, i_{9}: C, i_{10}: O, i_{11}: C, i_{12}: C\right\}$ are three different configurations for the topology depicted in Figure 1, with $C$ that indicates closed state and $O$ the open state. Among those, the last configuration is invalid as it would disconnect Cable 4 from the energy network, leading to disconnected customers. The configuration of the grid obviously affects the load of each cable. For instance, let us assume that the measured prosumption (of directly connected customers) equals $40 \mathrm{~A}$ in Cable 4 and $20 \mathrm{~A}$ in Cable 5 . The computed load for Cable 5 is $30 \mathrm{~A}$ for the first configuration and $60 \mathrm{~A}$ for the second (because $i_{7}$ is closed in this second configuration).

When the state of some fuses is uncertain, the actual configuration of the grid is not known. In turn, it entails uncertainty in the computed load of the cables, which, as the above example illustrated, is subject to significant variations. This raises the need to consider all configurations that may exist when performing such computations with their confidence level.

\section{EFFicient PRopagation OF UNCERTAINTY}

In this section, we describe the full process to propagate fuse states uncertainty to the approximated load. This process is an extension of the load approximation proposed by Hartman et al., [14]. It is composed of three steps. First, we detect uncertain fuses. Based on them, we list all valid configurations, and finally, we approximate the load.

\section{A. Business rules}

a) Rule 1: Disconnection of the grid: All customers of a grid with a non-null prosumption should be connected. For example, Cable 3 cannot be disconnected as it will disconnect customers directly linked to this cable and those connected on Cable 4 and 5. Consequently, a fuse cannot be open if it disconnects part of or the entire grid with non-null prosumption (Rule 1). Applying this rule to our example, $i_{5}$ cannot be open as it will disconnect all cables connected to Cabinet 2 and $3 . i_{7}$ can be open as it exists another power flow $\left(i_{9}, i_{1} 0, i_{8}\right)$ to connect Cable 4 to the transformer. From this rule, we experimentally derived five others (Rules 1.1 to 1.5). All rules should be valid for the part of the grid with non-null prosumption, or that feeds another part of the grid with non-null prosumption. We notice that they are equivalent to Rule 1 while being more efficient in executing during our experiments.

b) Rule 1.1: Mandatory power flow: We observe that some parts of the power flow remain identical for all valid configurations. This forms what we call the mandatory power flow. Therefore, all fuses that belong to a mandatory power flow are closed (Rule 1.1). Applying this rule on our example, we know that $i_{5}$ and $i_{6}$ should be closed. We can, thus, invalidate all configurations where at least one of these fuses is open. 
TABLE I

Business RULES

\begin{tabular}{|c|c|l|}
\hline $\begin{array}{c}\text { Rule } \\
\text { nb. }\end{array}$ & Area of application & \multicolumn{1}{c|}{ Description } \\
\hline 1 & All & A set of fuses cannot be open if they disconnect part of or the entire grid. \\
\hline 1.1 & Mandatory power flow & All fuses are closed. \\
1.2 & $\begin{array}{c}\text { Cable } \\
\text { Dead-end cable } \\
\text { Transformer }\end{array}$ & $\begin{array}{l}\text { The fuse that does not belong to the dead-end cabinet is closed. } \\
\text { At least one fuse should be closed. } \\
\text { If one cable has a measured prosumption different from zero, then exactly two fuses are open } \\
\text { if and only if they both belong to the same entity that can be a dead end. Otherwise, at most } \\
\text { one fuse of the circle is open. }\end{array}$ \\
1.4 & Circle &
\end{tabular}

c) Rule 1.2: Cable: A cable can be connected if and only if at least one of its fuses is closed. If both are open, then the cable cannot receive any power. Thus, Rule 1.2 can be formulated as follows: for all cables that have a measure prosumption different from zero, at least one of its fuses is closed. For example, Cable 4 can be connected if and only if the fuse $i_{7}$ or $i_{8}$ is closed.

d) Rule 1.3: Dead-end cable: Dead-end cables, like Cable 6 , connect dead-end cabinets, a cabinet with only one fuse. For all dead-end cables, the fuse that does not belong to the dead-end cabinet is closed (Rule 1.3). For example, fuse $i_{11}$ should be closed. If the fuse is open, the power flow will not be able to reach the dead-end cable.

e) Rule 1.4: Transformer: Transformers are the root entities of the low-voltage grid. If all fuses are open, then the power cannot be delivered to the grid. For example, fuse $i_{1}$ and $i_{3}$ cannot be open at the same time. For all transformers, at least one fuse should be closed (Rule 1.4).

f) Rule 1.5: Circle: Circles are formed either by two parallel cables, such as Cable 1 and Cable 2, or by indirectly parallel cables (not shown in our example). In this kind of topology, one can open one fuse without breaking the power flow. Opening more than one fuse breaks the power flow between the open fuses. For example, fuse $i_{7}$ can be open without disconnecting any cable while opening fuse $i_{7}$ and $i_{10}$ disconnects Cable 4 . However, one exception exists if a possible dead-end cabinet is part of the circle. A possible dead-end cabinet contains two fuses connected to only one other cabinet. For example, Cabinet 3 is a possible dead-end cabinet as it is only connected to Cabinet 2. Opening the two fuses of a possible dead-end cabinet does not break the power flow. For example, if $i_{8}$ and $i_{10}$ are open, the power flow can still go through $i_{7}$ to feed the Cable 4 and through $i_{9}$ to feed the Cable 5. Combining these two elements, the last rule can be formulated as follows: in a circle, exactly two fuses are open if and only if they both belong to a possible dead-end cabinet. Otherwise, at most one fuse of the circle is open (Rule 1.5).

\section{B. Inferring uncertain fuses}

The first step infers uncertain fuses by applying Rule 1.1. To perform that, we detect fuses that belong to the mandatory path as they must be closed. Therefore, the grid operator knows with the most thorough confidence what the states of these fuses are. In our example, if the measured power prosumption value is different from 0 on Cable 3, 4, or 5 , then fuses $i_{5}$ and $i_{6}$ are closed. This first step allows us to reduce the number of possible configurations. Removing $\mathrm{n}$ fuses removes $2^{n}$ possibilities. Therefore, this step is here to optimise the propagation.

Fuses that belong to the mandatory power flow depend on the topology. Thus, this step can be executed once, and the result can be saved. It becomes a tradeoff problem between execution time and memory consumption that we let engineers solve according to their requirements.

\section{Listing all valid configurations and their confidence}

We process circles independently to list all valid configurations. All possible configurations are, first, listed with their confidence level. It is done using the list of uncertain fuses computed in the previous step. Invalid ones are removed and their confidence levels are added to valid ones. Finally, we merge all the valid configurations of each circle to get the final list.

a) Computing the confidence level of a configuration: The confidence of one configuration (valid or not) corresponds to the intersection of the confidence of all fuse states. Let us assume that in our example, only $i_{1}, i_{4}$, and $i_{7}$ are uncertain. The grid operator knows that $i_{1}$ is closed with $79 \%$ confidence, that $i_{4}$ is closed with $82 \%$ confidence, and that $i_{7}$ is closed with $64 \%$ confidence. The confidence of the configuration that all fuses are closed equal to the intersection of the confidence that all fuses are closed. We consider that the states of fuses are independent and disjoint ${ }^{1}$. Following the probability theory, the confidence of this configuration equal $79 \% * 82 \% * 64 \%=41,46 \%$.

b) Listing valid configurations of circles: Experimentally, we observe that only fuses that belong to a circle can be uncertain. Moreover, circles can be considered as independent systems. The input ${ }^{2}$ and output ${ }^{3}$ loads do not depend on the configuration of fuses inside circles ${ }^{4}$. In our example, whatever the configuration is for fuses $\left\{i_{1}, i_{2}, i_{3}, i_{4}\right\}$, the load in fuse $i_{11}$ should correspond to the prosumption measured in Cable 6 . The load in fuse $i_{5}$

\footnotetext{
${ }^{1}$ The state of one fuse does not impact the state of another fuse

${ }^{2}$ Last fuse(s) before (in terms of power flow) the circle that get(s).

${ }^{3}$ First fuse(s) after the circle that get(s) the power.

${ }^{4}$ If the configuration is valid.
} 
should correspond to the prosumption measured in Cables 3,4 , and 5 . In our example, for the circle composed of $\left\{i_{1}, i_{2}, i_{3}, i_{4}\right\}$, there are $2^{2}=4$ possibilities since only fuses $i_{1}$ and $i_{4}$ are uncertain.: $\{(\mathrm{C}, \mathrm{C}) ;(\mathrm{C}, \mathrm{O}) ;(\mathrm{O}, \mathrm{C}) ;(\mathrm{O}, \mathrm{O})\}$.

Using rules defined in the previous sections, we remove all invalid configurations. We experimentally observe that when we remove an invalid configuration, we always replace it by the configuration with the maximal number of closed fuses. Therefore, we add the removed configuration's confidence level to the one with the most closed fuses.

c) Merging each possibilities for each circle: For each circle, there is a set of valid possibilities with their confidence level. The set of all valid configurations for the grid corresponds to the Cartesian products of these configurations. In our example, we compute the Cartesian product of the possible configurations of the first circle $(\{(\mathrm{C}, \mathrm{C}) ;(\mathrm{C}, \mathrm{O}) ;(\mathrm{O}, \mathrm{C})\})$ with the possible configurations of the second circle $(\{\mathrm{O}, \mathrm{C}\})$. The confidence level of a possible configuration for the entire grid corresponds to the intersection (and) of each circle's confidence level. As these confidence levels are independent and disjoint, it is computed by multiplying them.

\section{Approximating loads}

For each of the valid configuration retrieved from the previous step, we approximate the load. We apply the technique described in [14]. This gives us, for each possibility, the load of the fuse to which we attach the confidence of the configuration. Finally, for the cable load and for each configuration, we compute the fuses' maximal load, and we attached the confidence level of the configuration. For identical loads (cable or fuse), we merge the result, and we add the confidence level of all the different configurations.

\section{Experimental Setup}

\section{A. Research Questions}

Our work starts from the premise that uncertainty can significantly affect the computed grid properties. As such, our first research question concerns the quantitative evaluation of this impact, particularly cable load estimation. That is, we ask how different could be the estimated load of a given cable in two alternative grid configurations (resulting from the uncertainty of some fuses). We ask: (RQ1) how much do estimations of cable loads vary in different grid configurations?

To answer this question, we consider a real-world grid topology from the Luxembourgish power grid, provided by our industrial partner. Starting from the assumption that all fuses are uncertain, we estimate each cable's load under each topology's valid configuration. Then we measure, for each cable, the median and standard deviation of its estimated load in all configurations. A higher deviation demonstrates a more significant impact of uncertainty on the resulting cable loads. Given that energy production and consumption determine cable load (and, thus, may affect our conclusion), we repeat this process 10,000 times, using each time different measured consumption data.

Having shown that uncertainty can indeed lead to variations in estimated cable load, we turn our attention towards the factors which may poison (introduce undesired noise in) these computations. In particular, we want to assess whether invalid configurations can significantly impact the results when not filtered out. Thus, we ask: (RQ2) Do invalid configuration significantly alter the cable load estimations? To address this question, we compute the standard deviation of our real-world topology's cable load in two cases: considering valid configuration only and considering all configurations (including invalid ones). Different standard deviations would indicate that invalid configurations alter cable load differently than valid ones.

An additional motivation to removing invalid configurations is to reduce computation time (by reducing the number of configurations for which one estimates cable load). Our third and last research question evaluates this benefit. We ask: (RQ3) What is the performance gained by filtering out invalid grid configurations? We evaluate this reduction by first measuring the proportion of invalid configurations, and then quantitatively (by measuring the actual computation time).

\section{B. Implementation and Replication Package}

We implemented our approach in a prototype tool named SimSG. It is composed of a web user interface (in JavaScript) and a backend (in Java). Both are publicly available on Bitbucket ${ }^{5}$. To estimate cable loads, we reuse the approach and prototype of [14]. We also provide the implementation of our experiments in a separate replication package. ${ }^{6}$ Regarding the performance evaluation, we use the state-of-the-art framework for microbenchmarking: $\mathrm{JMH}^{7}$. We executed all our experiments on a MacBook Pro 2016 with an Intel Core i7-6700HQ 2.6 $\mathrm{GHz}$ processor and $16 \mathrm{~GB}$ of RAM.

\section{Results}

\section{A. RQ1: Impact of uncertainty on load estimation}

Figure 2 shows, for each cable, the standard deviation (in amps) of the estimated cable load across valid grid configurations, observed under different consumption values. We notice that the remaining cables $1,3,11,12,13,14$, and 15 exhibit a standard deviation of zero, regardless of the consumption values. This is because these cables are part of the mandatory power flow, and thus their load is not impacted by the grid configuration.

On the contrary, cables 2 and 4 to 10 exhibit standard deviations greater than zero. Knowing that a cable load typically ranges from 0 to $200 \mathrm{amps}$, this means that their

\footnotetext{
${ }^{5}$ https://bitbucket.org/sntcreos/simsg-website, https://bitbucket.org/sntcreos/simsg-java

${ }^{6}$ https://bitbucket.org/sntcreos/bench-simsg-java

${ }^{7}$ https://openjdk.java.net/projects/code-tools/jmh/
} 


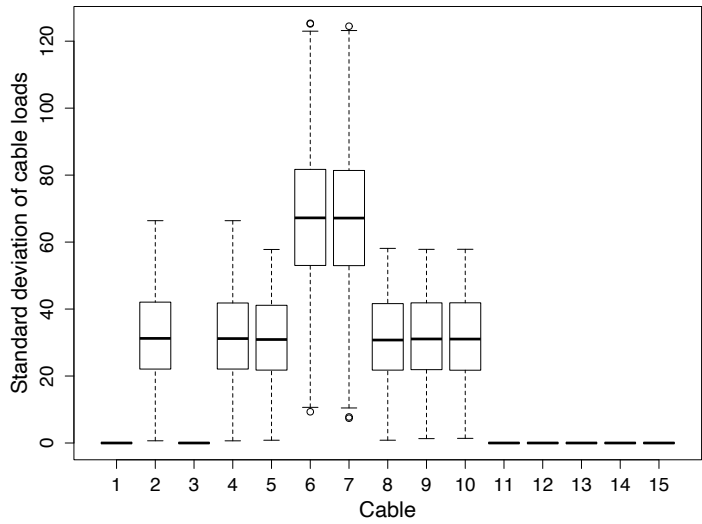

Fig. 2. Standard deviations (in amps) of the estimated cable load across valid grid configurations. Each data point of a box plot represents this standard deviation at a particular point in time (that is, each relates to different consumption values).

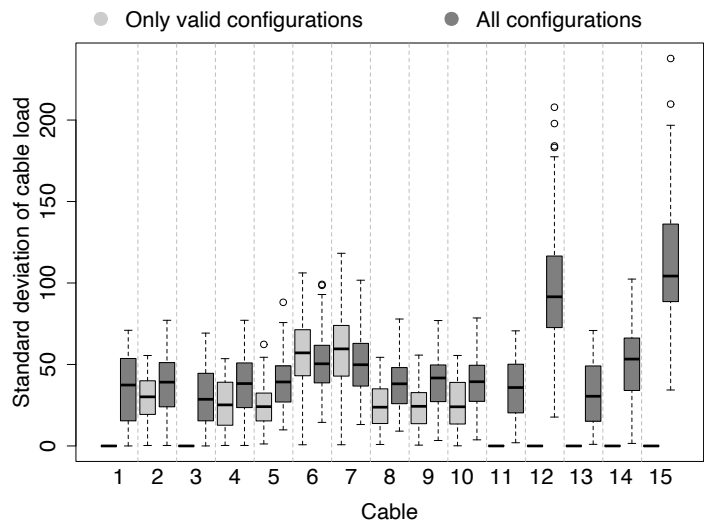

Fig. 3. Comparison of the standard deviation in cable load when one considers all configurations (dark gray) or only valid configurations (light gray).

estimated load varies across the different grid configurations. Cables 6 and 7 show the highest deviations. These cables belong to the same circle, and they are not a deadend of the topology. Thus, it appears that the closer to the transformation a circle a cable is, the larger the standard deviation of his load will be. Indeed, a cable in a circle may contain the load to feed all customers of the circle plus all customers after (in the power flow), or just the customers' load connected to this cable. A detailed look at our results reveals that, between two configurations, the load of cables $2,4,8$, and 9 can up to double, whereas, for cables 6 and 7 , the load can be multiplied by a maximum factor of 4 . Overall, this shows that uncertainty in the grid configuration can lead to drastic changes in the estimated cable loads.

\section{B. RQ2: Load estimations within invalid configurations}

Figure 3 shows the standard deviation in cable load when all or only valid configurations are considered. As before, a distinction has to be made between cables that are part of the mandatory power flow and those that are not. Interestingly, we observe that invalid configurations introduce variance in the former category's estimated load, while there are none when only valid configurations are considered. This is because invalid configurations do not comply with the property that those cables (belonging to the main power flow) receive any power flowing through the grid. As for the other cables, a Mann-Whitney U test reveals that the differences in standard deviations are statistically significant, with a p-value smaller than $10^{4}$. This means that invalid configurations significantly affect the variation of the cable load and should be disregarded.

\section{RQ3: Performance}

Figure 4 shows the performance gain achieved by filtering out invalid configurations. More precisely, Figure 4a shows the ratio of valid configurations, considering that different numbers of fuses are uncertain (configurable) while the other fuses have a known status (open or close). We observe that this ratio diminishes as the number of uncertain fuses increases. Thus, a higher uncertainty entails a higher amount of wasted computation resources (since invalid configurations are relatively more common than valid ones). Combined with our RQ2 results, higher uncertainty also induces a higher impact of invalid configurations on the cable load estimation results.

We show the actual performance impact in Figure $4 \mathrm{~b}$. Therein, "naive" refers to cable load estimation when considering all grid configurations (including invalid ones), whereas "with rules" refers to the estimation made on valid configurations only added to the time required to execute our validity rules. The overhead of executing our rules is offset as soon as three fuses on the grid are uncertain. More generally, the performance gain increases exponentially with the number of uncertain fuses, which is also explained by the decreasing ratio of valid configurations. When all fuses are uncertain, filtering out the valid configurations reduces the total computation time by three orders of magnitude (that is, more than 1,000).

\section{Threats to Validity}

The main threat to the validity of our conclusions comes from the selection of our case study. Although taken from the real world, the results presented are limited to this particular topology. To improve confidence in the generality of our conclusions, we replicated our experiments on two other real-world grid topologies (provided by our partner) and 100 randomly generated topologies. Each of these generated topologies comprises one substation and ten cabinets (this ratio represents the power grid in Luxembourg). Between the two cabinets, we randomly put one or two cables.

It turned out that these new experiments led to the same conclusions presented above. That is: (1) uncertainty can profoundly affect the results of computing cable load estimations; (2) invalid configurations introduce significant noise in those computations; (3) discarding those invalid configurations yields a substantial performance gain. 


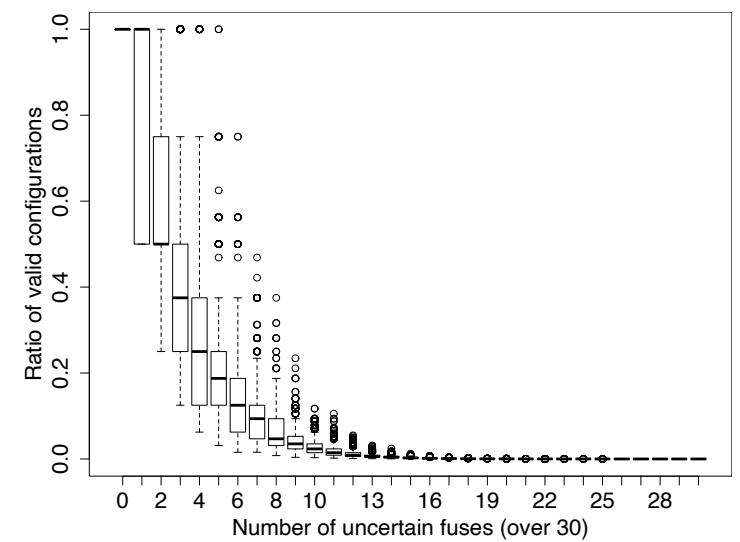

(a) Ratio of valid configurations wrt. the number of uncertain fuses

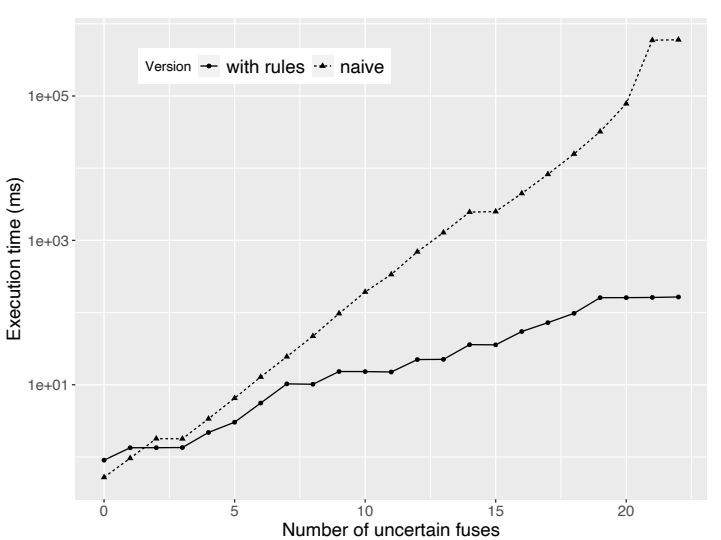

(b) Performance comparison for cable load estimation

Fig. 4. Performance gained by filtering out invalid configurations

\section{Conclusion AND Future WORK}

In this paper, we presented an approach to efficiently and validly propagate fuse states' uncertainty through the load approximation. To perform that, we first defined a set of business rules, all the derived version of a more general one. These rules serve to validate or invalidate the different possible grid configurations. In addition, we define a process to propagate the confidence level from fuse states to approximated load (cable or fuses). We validated the rules and evaluated the process using a topology from the power grid in Luxembourg. We show that our approach outperforms the naive version due to the ratio of valid configurations over the total number of configurations.

In this work, we focus on isolated transformers. That is a simplification of what is happening in real topologies. As future work, we will need to add new rules to consider cables that (indirectly) connect two transformers. Knowing that, in the Luxembourgish power grid, the operator avoids connecting two transformers as it makes the load computation (or approximation) more complex. And it could reduce the quality of his knowledge of the effective situation. Moreover, we did this work in close relation with the grid operator. We let for next steps the evaluation of applicability over grid networks by using, for example, the IEEE use case. Finally, in this study, we hypothesise that fuse states' confidence is given, and it is computed as independent events. Future work should focus on how to estimate the fuse states' confidence level.

\section{ACKNOWLEDGMENTS}

We would like to thank Yves Reckinger and Robert Graglia from Creos S.A. for their insightful feedback.

\section{REFERENCES}

[1] A. Zidan and E. F. El-Saadany, "A cooperative multiagent framework for self-healing mechanisms in distribution systems," IEEE Trans. Smart Grid, vol. 3, no. 3, pp. 1525-1539, 2012.
[2] N. Antoniadis, M. Cordy, A. Sifaleras, and Y. L. Traon, "Preventing overloading incidents on smart grids: A multiobjective combinatorial optimization approach," in $O L A$, vol. 1173 of Communications in Computer and Information Science, pp. 269-281, Springer, 2020.

[3] K. Shahid, E. Schiavone, D. Drenjanac, M. Lyhne, R. L. Olsen, and H. Schwefel, "Handling incomplete and erroneous grid topology information for low voltage grid observability," in SmartGridComm, pp. 1-6, IEEE, 2019.

[4] B. Borkowska, "Probabilistic load flow," IEEE Transactions on Power Apparatus and Systems, vol. PAS-93, no. 3, pp. 752-759, 1974.

[5] J. F. Dopazo, O. A. Klitin, and A. M. Sasson, "Stochastic load flows," IEEE Transactions on Power Apparatus and Systems, vol. 94, no. 2, pp. 299-309, 1975

[6] M. Madrigal, K. Ponnambalam, and V. H. Quintana, "Probabilistic optimal power flow," in Conference Proceedings. IEEE Canadian Conference on Electrical and Computer Engineering (Cat. No.98TH8341), vol. 1, pp. 385-388 vol.1, 1998.

[7] Z. Q. Xie, T. Y. Ji, M. S. Li, and Q. H. Wu, "Quasi-monte carlo based probabilistic optimal power flow considering the correlation of wind speeds using copula function," IEEE Transactions on Power Systems, vol. 33, no. 2, pp. 2239-2247, 2018.

[8] W. Sun and M. Zamani, "A nonparametric bayesian approach for probabilistic representation of power uncertainties," in SmartGridComm, pp. 1-6, IEEE, 2019.

[9] J. M. Morales, L. Baringo, A. J. Conejo, and R. Minguez, "Probabilistic power flow with correlated wind sources," IET Generation, Transmission Distribution, vol. 4, no. 5, pp. 641$651,2010$.

[10] H. Schwefel, J. G. Rasmussen, R. L. Olsen, H. Ringgaard, and N. Silva, "Using smart meter measurements to manage accuracy of current calculations in lv feeders," in 2019 IEEE International Conference on Communications, Control, and Computing Technologies for Smart Grids (SmartGridComm), pp. 1-7, 2019.

[11] R. N. Allan, A. m. L. Da Silva, and R. C. Burchett, "Evaluation methods and accuracy in probabilistic load flow solutions," IEEE Transactions on Power Apparatus and Systems, vol. PAS100 , no. 5, pp. 2539-2546, 1981.

[12] V. Miranda, M. A. Matos, and J. T. Saraiva, "Fuzzy load flow - new algorithms incorporating uncertain generation and load representation," in Proc. Tenth Power System Computation Conf., Graz, Austria, pp. 621-627, 1990.

[13] C. Walck, "Hand-book on statistical distributions for experimentalists," tech. rep., University of Stockholm, 1996.

[14] T. Hartmann, A. Moawad, F. Fouquet, Y. Reckinger, J. Klein, and Y. L. Traon, "Near real-time electric load approximation in low voltage cables of smart grids with models@run.time," in SAC, pp. 2119-2126, ACM, 2016. 